\title{
Comprehensive investigation of the gene expression system regulated by an Aspergillus oryzae transcription factor XInR using integrated mining of gSELEX- Seq and microarray data
}

\author{
Hiroya Oka ${ }^{1}$, Takaaki Kojima ${ }^{1 *}$ (D, Kunio Ihara ${ }^{2}$, Tetsuo Kobayashi ${ }^{1}$ and Hideo Nakano ${ }^{1}$
}

\begin{abstract}
Background: Transcription factors (TFs) specifically bind to DNA sequences and control the expression of target genes. AoXInR is a key TF involved in the expression of xylanolytic and cellulolytic enzymes in the filamentous fungi, Aspergillus oryzae. Genomic SELEX-Seq (gSELEX-Seq) can reveal the in vitro binding sites of a TF in a genome. To date, the gene expression network controlled by AoXInR in A. oryzae is not fully explored. In this study, the data from gSELEX-Seq analysis and data mining were applied toward a comprehensive investigation of the AoXInR-regulated transcriptional network in A. oryzae.

Results: Around 2000 promoters were selected as AoXInR-binding DNAs using gSELEX-Seq, consequently identifying the genes downstream of them. On the other hand, 72 differentially expressed genes (DEGs) related to AoXInR had been determined by microarray analysis. The intersecting set of genes, that were found using the gSELEX-Seq and the microarray analysis, had 51 genes. Further, the canonical AoXInR-binding motifs, 5'-GGCT(A/G) A-3', were successfully identified in gSELEX-Seq. The motif numbers in each promoter of the DEGs and differential expression levels were correlated by in silico analysis. The analysis showed that the presence of both 5'-GGCTAA-3' and 5'-GGCTGA-3' motif has significantly high correlation with the differential expression levels of the genes.

Conclusions: Genes regulated directly by AoXInR were identified by integrated mining of data obtained from gSELEX-Seq and microarray. The data mining of the promoters of differentially expressed genes revealed the close relation between the presence of the AoXInR-binding motifs and the expression levels of the downstream genes. The knowledge obtained in this study can contribute greatly to the elucidation of AoXInR-mediated cellulose and xylan metabolic network in A. onyzae. The pipeline, which is based on integrated mining of data consisting of both in vitro characterization of the DNA-binding sites and TF phenotype, can be a robust platform for comprehensive analysis of the gene expression network via the TFs.
\end{abstract}

Keywords: Aspergillus oryzae, gSELEX-Seq (genomic SELEX-Seq), XInR, Transcriptome

\footnotetext{
* Correspondence: kojimat@agr.nagoya-u.ac.jp

${ }^{1}$ Department of Applied Biosciences, Graduate School of Bioagricultural

Sciences, Nagoya University, Furo-cho, Chikusa-ku, Nagoya 464-8601, Japan

Full list of author information is available at the end of the article
}

(c) The Author(s). 2019 Open Access This article is distributed under the terms of the Creative Commons Attribution 4.0 International License (http://creativecommons.org/licenses/by/4.0/), which permits unrestricted use, distribution, and reproduction in any medium, provided you give appropriate credit to the original author(s) and the source, provide a link to the Creative Commons license, and indicate if changes were made. The Creative Commons Public Domain Dedication waiver (http://creativecommons.org/publicdomain/zero/1.0/) applies to the data made available in this article, unless otherwise stated. 


\section{Background}

Transcription factors (TFs) interact with the enhancers and promoters on a genome and play a central role in transcription regulation in cells [1]. Identifying the genes regulated by a TF is important for understanding not only the function of the TF but also the gene expression network of the cells. However, since laborious efforts are required to comprehensively identify TF-regulated genes, except for some model TFs, the detailed functions of most TFs are still poorly understood [2, 3]. Therefore, so far, various methods have been devised for high-throughput identification of genes regulated by a target TF.

DNA microarray (DNA-chip) analysis, which is a comprehensive DNA hybridization technique, was proposed by Brawn et al. [4]. This technique is mainly applied for simultaneous analysis of the expression of more than thousands of genes. To date, many genes regulated by TFs have been identified using this method in various organisms $[5,6]$. However, since DNA microarray analysis gives no information of the TF-binding sites, it is very difficult to distinguish whether the expression of the differentially expressed genes (DEGs) is regulated directly or indirectly by a target TF.

On the other hand, systematic evolution of ligands by exponential enrichment (SELEX) is a method used for selecting nucleotides that specifically bind to a target from a random sequence pool in vitro [7-9]. Genomic SELEX (gSELEX), in which the binding sites of a target TF can be preferentially enriched from a genomic library in vitro, enables direct mapping of the TF-binding sites within the genome [10]. Dror et al. showed that the form of DNA near the consensus motif contributes to the determinant of the TF-binding sequence [11]. The study suggested that it is important to use genomic libraries rather than synthetic DNA libraries for the identification of TF-binding sequences. Recently, Kojima et al. established a new comprehensive analysis system for transcriptional regulatory networks by using gSELEX-Seq and RNA-Seq [12]. This system identified the genes directly regulated by AmyR, a TF from A. nidulans [12]. The analytical pipeline is a robust platform for comprehensive genome-wide identification of genes regulated by a target TF.

$A$. oryzae is one of the most widely used industrial microorganisms in food production and brewing since ancient times in Asia. Currently, this fungus is expected to have further industrial applications such as the production of useful proteins or metabolites, not only because of its high protein-production ability but also the safety for human health and the environment [13]. In addition, since a variety of cellulolytic and xylanolytic enzymes can be produced in $A$. oryzae, this is an attractive microorganism for biofuel production from plant biomass, which is mainly composed of cellulose, hemicelluloses, and lignin [14]. To utilize the xylanolytic or cellulolytic pathway of $A$. oryzae for such applications, it is extremely important to understand the transcriptional network controlled by TFs.

Filamentous fungi can produce various enzymes in the presence of cellulose or $\beta-1,4$ xylan. XlnR has been identified as a TF involved in the expressions of xylanolytic and cellulolytic enzymes in Aspergillus niger [15-18]. The XlnR (AnXlnR) binds to 5'-GGCTAAA-3' and $5^{\prime}$-GGCTAG-3' via the $\mathrm{Zn}(\mathrm{II})_{2} \mathrm{Cys}_{6}$ zinc finger motif $[17,19]$. AoXlnR, an ortholog of $X \ln R$ in $A$. oryzae, also controls the expression of xylanolytic and cellulolytic genes [20-22]. The binding sites of AoXlnR have been presumed to be 5'-GGCTAA-3' and 5'-GGCTGA-3' by electrophoretic mobility shift assay (EMSA) using AoXlnR [20-22].

In this study, the genes directly regulated by AoXlnR were successfully identified by combining data obtained from gSELEX-Seq and microarray analyses [22]. The AoXlnR-binding motifs extracted from the selected DNA sequences by gSELEX-Seq were consistent with the canonical motifs reported in the previous study [21]. Furthermore, correlations between the motifs in promoters of the DEGs and the differential expression levels were analyzed by using bioinformatics data mining. Finally, the results obtained from the comprehensive analysis in this study are discussed.

\section{Methods}

\section{Construction of an $A$. oryzae genomic library}

$A$. oryzae genomic library was constructed as described previously [12], with some modifications. A wild type $A$. oryzae strain, RIB40 (National Research Institute of Brewing Stock Culture ATCC-42149) was used to isolate genomic DNA in this study. Linkers were prepared by annealing the corresponding primer pairs: Nextra-Read1/ Nextra-Read1-adp-comp and Nextra-Read2/Nextra-Read2-adp-comp (Additional file 1: Table S1). One nanogram of genomic DNA that was size-fractionated to approximately $100 \mathrm{bp}$ was amplified in a $20-\mu \mathrm{L}$ PCR mixture containing 0.025 U/ $\mu \mathrm{L}$ LA Taq (Takara Bio, Otsu, Japan) and $0.25 \mu \mathrm{M}$ of each of the primers Nextra-Read1 and Nextra-Read2 (Additional file 1: Table S1) in the following PCR program: preheating at $94^{\circ} \mathrm{C}$ for $3 \mathrm{~min} ; 15$ cycles consisting of $94^{\circ} \mathrm{C}$ for $15 \mathrm{~s}$ and $68^{\circ} \mathrm{C}$ for $2 \mathrm{~min}$; and additional extension at $72{ }^{\circ} \mathrm{C}$ for $7 \mathrm{~min}$. The amplicons were purified using MinElute PCR Purification Kit (Qiagen, Tokyo, Japan) for gSELEX analysis.

\section{Expression of recombinant AoXInR in Escherichia coli}

The N-terminal 183 amino acids of AoXlnR that included the DNA-binding domain was expressed as a MalE (maltose-binding protein $[\mathrm{MBP}]$ ) fusion protein in 
E. coli BL21(DE3) [12, 21], followed by purification according to the procedure described in a previous study [23]. The purified protein concentration was determined with the Bradford assay, with bovine serum albumin (BSA) as a standard [24].

\section{In vitro selection of AoXInR-bound DNA fragments from} the $A$. oryzae genomic library using gSELEX-Seq gSELEX was performed according to the procedure described in a previous study [12], with some modifications. In this study, $20 \mathrm{ng}$ of $A$. oryzae genomic library was mixed with $100 \mu \mathrm{L}$ of $0.4 \mu \mathrm{M}$ purified $\mathrm{MBP}-\mathrm{X} \ln \mathrm{R}_{1-}$ 183 in PBS $(137 \mathrm{mM} \mathrm{NaCl}, 2.7 \mathrm{mM} \mathrm{KCl}, 10 \mathrm{mM}$ $\mathrm{Na}_{2} \mathrm{HPO}_{4}, 1.8 \mathrm{mM} \mathrm{KH} \mathrm{PO}_{4}, \mathrm{pH}$ 7.4) containing $10 \mathrm{mM}$ 2-mercaptoethanol (PBS/2-ME) and agitated for $30 \mathrm{~min}$ at room temperature. Next, $10 \mu \mathrm{L}$ of amylose resin (New England BioLabs, Ipswich, MA, USA) was washed with $500 \mu \mathrm{L}$ of MBP without (w/o) EDTA buffer $(200 \mathrm{mM}$ $\mathrm{NaCl}, 20 \mathrm{mM}$ Tris-HCl, $10 \mathrm{mM}$ 2-mercaptoethanol, $\mathrm{pH}$ 7.5 ) and then, the resin was suspended in a $1.5-\mathrm{mL}$ tube in $900 \mu \mathrm{L}$ of fresh MBP w/o EDTA buffer and mixed with the $\mathrm{MBP}-\mathrm{Xln} \mathrm{R}_{1-183}$-binding reaction mixture. The suspension was mixed using a rotator for $1 \mathrm{~h}$ at $4{ }^{\circ} \mathrm{C}$, following which the resin was recovered by centrifuging the suspension at $300 \times g$ for $1 \mathrm{~min}$ at $4{ }^{\circ} \mathrm{C}$. The MBP-XlnR $1-$

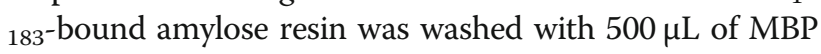
w/o EDTA buffer. After removing as much of the supernatant as possible, the resin was suspended in $10 \mu \mathrm{L}$ of MBP w/o EDTA elution buffer $(200 \mathrm{mM} \mathrm{NaCl}, 20 \mathrm{mM}$ Tris- $\mathrm{HCl}, 10 \mathrm{mM}$ 2-mercaptoethanol, $20 \mathrm{mM}$ maltose, $\mathrm{pH} 7.5$ ), and the suspension was mixed using a rotator for $15 \mathrm{~min}$ at $4^{\circ} \mathrm{C}$. Lastly, the supernatant was recovered after centrifugation at $300 \times g$ for $1 \mathrm{~min}$ at $4{ }^{\circ} \mathrm{C}$.

The selected DNA fragments in the supernatant were amplified using a PCR mixture (8 tubes $\times 20 \mu \mathrm{L})$ that included $0.025 \mathrm{U} / \mu \mathrm{L}$ LA Taq DNA polymerase (Takara) and $0.25 \mu \mathrm{M}$ primers (Nextra-Read1 and Nextra-Read2) in the following program: preheating at $95^{\circ} \mathrm{C}$ for $3 \mathrm{~min}$; 14 cycles (in the first round), 12 cycles (in the second round), or 10 cycles (in the third round) of $94{ }^{\circ} \mathrm{C}$ for $15 \mathrm{~s}$ and $68^{\circ} \mathrm{C}$ for $2 \mathrm{~min}$; and an additional extension at $72^{\circ} \mathrm{C}$ for $7 \mathrm{~min}$.

\section{Analysis of relative AoXInR-binding affinity by using bead display and flow cytometry}

The selected DNA fragments from gSELEX (from each round) were PCR-amplified using the primers Nextra-Read1-bio and Nextra-Read2-Cy5, followed by purification using MinElute PCR Purification Kit for the immobilization of M-280 streptavidin-coated beads (Dynabeads M-280 Streptavidin; Life Technologies, Carlsbad, CA, USA). The relative AoXlnR-binding activity of the fragments in each pool selected by gSELEX was examined by using flow cytometry (JSAN; Bay
Bioscience, Kobe, Japan) as described by Kojima et al. [12]. The flow cytometric data were analyzed by using the FlowJo software (Treestar, Ashland, OR, USA).

\section{DNA sequencing and data analysis in gSELEX-Seq}

For sequencing with an Illumina MiSeq sequencer (Illumina, San Diego, CA, USA), the selected DNA fragments by gSELEX (from each round) were purified using the Agencourt AMPure XP system (Beckman Coulter, Brea, CA, USA). All sequencing data are available under controlled access through the DNA Databank of Japan (DDBJ; accession number DRA006473).

The $5^{\prime}$ and $3^{\prime}$ adapters were stripped from the reads by using Cutadapt (v1.7.1, https://cutadapt.readthedocs.io/en/ stable/\#) with the following parameters: -g TCGTCGGCA GCGTCAGATGTGTATAAGAGACAG -a CTGTCTCTT ATACACATCTGACGCTGCCGACGATT -g GTCTCGT GGGCTCGGAGATGTGTATAAGAGACAG -a CTGT CTCTTATACACATCTCCGAGCCCACGAGACTT -O 15. The trimmed paired-end reads were mapped with Bowtie (v2) onto the $A$. oryzae genome sequences (A_oryzae_RIB40_current_chromosomes_13_Mar_2016 [25]) with default settings. Peaks were called using MACS (v1.4.2) with default settings, except for the following options: -f BAM -g 40,000,000. Once the peaks were ranked on the basis of fold enrichment, the peak interval data were converted to that of 50-bp sequences, which were cut out in each direction from the summit position by using BEDTools (v2.17.0) with the following parameters: bedtools slop -1 24 -r 24. The sequence data were extracted using the fastaFromBed utility in BEDTools.

Candidate promoters regulated by AoXlnR were annotated as follows: The A oryzae upstream 1000 dataset, which contains the 1000-bp region upstream of all of the predicted A. oryzae genes, was obtained using A_oryzaeRIB40_current_orf_genomic_1000.fasta [25]. The 50-bp sequences obtained from the third round of selection were annotated by local BLAST in A. oryzae upstream1000 with the following parameters: blastn -evalue 10 -outfmt 6. Subsequently, motifs were identified by MEME ( $v$ 4.10.2) with the following parameters: -dna -maxsize 500,000 -nmotifs 5 -revcomp -maxw 20.

\section{Affinity determination for MBP-AoXInR against AoXInR- binding fragments by using biolayer interferometry (BLI)} The XRE-WT fragment (the region between - 179 to $93 \mathrm{bp}$ from the initiation codon of AO090103000423 [xynF1], an AoXlnR-binding fragment retaining two AoXlnR-binding motifs reported in the previous study [21]), was amplified from A. oryzae genomic DNA with $0.25 \mu \mathrm{M}$ of each of the primers XRE-S-bio and XRE-AS (Additional file 1: Table S1). xynF1_upstream _1 (the region between -470 to $-380 \mathrm{bp}$ from the initiation codon of $x y n F 1$ ) was amplified from A. oryzae genomic 
DNA with $0.25 \mu \mathrm{M}$ each of xynF1-F-bio and xynF1-R (Additional file 2: Figure S1A and Additional file 1: Table S1). Double-stranded egl-242 (the region between -781 to $-727 \mathrm{bp}$ from the initiation codon of AO09 0023000787), egl-363 (the region between -660 to -607 bp from the initiation codon of AO090023000787), egl-617 (the region between -406 to $-356 \mathrm{bp}$ from the initiation codon of AO090023000787), abf-687 (the region between -344 to $-295 \mathrm{bp}$ from the initiation codon of AO090701000885), or abf- 830 (the region between -187 to $-139 \mathrm{bp}$ from the initiation codon of AO090 701000885) was prepared with bio-polyA as a complementary oligonucleotide by using Klenow fragments (Takara; Additional file 2: Figure S1B, C; Additional file 1: Table S1). All the biotinylated fragments were purified using MinElute PCR Purification Kit for BLI analysis.

The BLI is an optical analytical technique for monitoring biomolecular interactions utilizing the interference pattern of white light as it is reflected from a layer of immobilized biomolecules versus an internal reference layer [26]. The affinities of MBP-AoXlnR against several AoXlnR-binding fragments in the AO090103000423 (xynF1), AO090023000787, or AO090701000885 promoter region were determined by using the BLI on a BLItz system equipped with streptavidin sensors (Pall-ForteBio, Menlo Park, CA). A baseline was initially established in Kinetics Buffer 10× (Pall-ForteBio; 30 s). Then, $4 \mu \mathrm{L}$ of each biotinylated ligand $(500 \mathrm{nM}$ in Kinetics Buffer 10x) was captured (2 min) and a baseline was re-established in Kinetics Buffer $10 \times(30 \mathrm{~s})$. To analyze the affinity between MBP-AoXlnR and the immobilized ligand, $4 \mu \mathrm{L}$ of the given $\mathrm{MBP}-\mathrm{XlnR}_{1-183}$ was captured ( $2 \mathrm{~min})$, and the bound analyte was dissociated in $200 \mu \mathrm{L}$ of Kinetics Buffer 10× (3 min). AoXlnR was diluted with Kinetics Buffer $10 \times$ to the desired concentration $(0,25,50$, or $100 \mathrm{nM}$ for AoXlnR-binding fragments in the $x y n F 1 ; 0,100,200$, or $500 \mathrm{nM}$ for those in AO090023000787, or AO090701000885). All measurements were performed at room temperature with agitation. Data analysis and fitting were performed by using global fitting mode in a 1:1 binding model.

\section{Statistical analysis of AoXInR-binding sequence}

Correlations between $H X \ln R / \Delta X \ln R$ ratio for each expression level (i.e., differential gene expression between AoXlnR-overproducing and AoXlnR-disrupted $A$. oryzae strains [22]) and several parameters, which possibly affect the binding of AoXlnR, were analyzed using Spearman's rank correlation test and Pearson's correlation test in $\mathrm{R}$ software. Here, the parameters examined were as follows; the AoXlnR-binding motif numbers in the promoters of the DEGs, the fold enrichment values of and the summit position of the detected peaks from the selection round 3 in gSELEX (Additional file 3: Table S2).

\section{Results \\ In vitro selection of AoXInR-binding DNAs using gSELEX-Seq for identification of the candidate promoters regulated by AoXInR}

First, for performing gSELEX-Seq, the $A$. oryzae genomic DNA was fragmented to approximately less than $100 \mathrm{bp}$, ligated with linkers at both ends, and amplified using PCR (Additional file 4: Figure S2). Next, the DNA fragments binding to $M B P-X \ln R_{1-183}$, the MBP-fused AoXlnR DNA-binding domain, were selected from genomic library by three rounds of gSELEX. The DNA pools selected in each round of gSELEX were amplified with biotin and Cy5-labeled primers and then immobilized onto streptavidin-coated beads. Each bead pool was incubated with MBP-XlnR $\mathrm{R}_{1-183}$ followed by immunostaining with a fluorescein-labeled anti-MBP antibody. The bead complexes were analyzed by flow cytometry to monitor the progress of the gSELEX selection process (Additional file 5: Figure S3A). The relative fluorescein intensity increased with each round of selection. This result suggests that the DNA pool binding to AoXlnR was highly enriched in each round from the $A$. oryzae genomic library in gSELEX. Even in selection round 3 , an increase in the relative binding activity was observed (Additional file 5: Figure $\mathrm{S} 3 \mathrm{~B})$. In our previous approach using AmyR from $A$. nidulans (AnAmyR), saturation of the enrichment of the bound fragments was observed in selection round 3 of gSELEX analysis [12]. This increased enrichment efficiency might be caused by more appropriate selection conditions than previous one. It should be noted here that we used purified MBP-AoXlnR $R_{1-183}$ and not crude protein solution.

All the DNA pools selected from the genomic library were sequenced using an Illumina MiSeq system, followed by bioinformatics analysis for genome-wide identification of AoXlnR-binding sites. After the mapping of the sequenced tags onto the A. oryzae genome and the detection of peaks with high numbers of the mapped tags, 50-bp DNA fragment around the top of the peaks were cut out.

Each 50-bp tag from round 3 was annotated by local BLAST with the $A$. oryzae upstream 1000 dataset. Based on the annotation, we successfully identified 1948 promoters, including those of eight genes under the control of AoXlnR (xynF1, xynG1, xynG2, xylA, celA, celB, celC, and celD) [22] (Additional file 6: Table S3). This result reinforces the robustness of the genome-wide identification system for TF-regulated promoters using gSELEX-Seq. 
Identification of the genes regulated directly by AoXInR In order to identify AoXlnR targets, Noguchi et al. carried out DNA microarray analysis in which the data based on spots of the scanned microarray images were analyzed for measure the expression level in each gene according to the procedure reported by Tamano et al. [22, 27]. By the DNA microarray analysis, 75 DEGs that showed more than five-fold greater expression in the AoXlnR overproducer than in the disruptant were identified [22]. From these 75 genes, AO090003001341, AO090 701000828, and AO090005000768 were removed from the gene ID list in AspGD based on the current data of gene information for $A$. oryzae. The remaining 72 DEGs were compared with the 1948 genes possibly regulated by AoXlnR obtained from gSELEX-Seq; consequently, an intersecting subset had 51 genes (Fig. 1, Additional files 3 and 6: Table S2 and S3). This subset includes not only the aforementioned eight genes regulated directly by AoXlnR but also xylanolytic genes (AO090001000208, AO090005000698, and AO090 011000140) and glycolytic genes (AO090701000274, AO090103000087, and AO090012000445). Remarkably, the percentage of promoters containing the canonical binding motifs (5'-GGCTA/GA-3') in the 51 DEGs (86.3\% [44/51]), was significantly higher than that in the other 21 DEGs $(57.1 \%$ [12/21]; $p<0.05$ by Fisher's Exact Test). Generally, the genes identified as DEGs in microarray analysis should include ones regulated primarily or secondarily by the TFs. Together, the expressions of the 51 DEGs downstream of the promoters are considered to be directly controlled by AoXlnR. On the other hand, the other 21 DEGs, which were not included among the genes downstream of the candidate promoters, are considered to be indirectly regulated by AoXlnR.

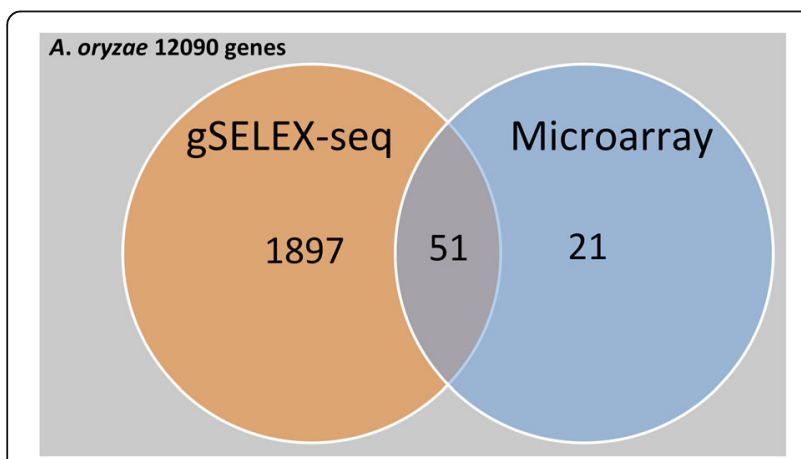

Fig. 1 Venn diagram of the numbers of XInR-related genes from gSELEX and microarray analyses. gSELEX-Seq: genes under the control of candidate AoXInR-regulated promoters, obtained using gSELEX; Microarray: DEGs that showed more than five-fold higher expression in the AoXInR overproducer than in the disruptant, which were identified using microarray analysis [22]. Values indicate the total number of genes in each set de novo motif analysis for identification of AoXInRbinding sites

de novo motif analysis of AoXlnR-binding sites was performed using the extracted 50-bp tags from gSELEX-Seq analysis (Fig. 2). The results revealed that $5^{\prime}$-(c/a) GGcT $(\mathrm{A} / \mathrm{g})(\mathrm{A} / \mathrm{t})(\mathrm{a} / \mathrm{t})-3^{\prime}$ was clearly detected as an AoXlnRbinding motif in all selection rounds. The binding motif contained one of the major canonical XlnR-binding motif, 5'-GGCTAA-3' [21]. It should be noted that guanine was also detected at a relatively high frequency at the fifth base in the motif. This result is quite reasonable because AoXlnR binds the 5'-GGCTGA-3' sequence with lower binding affinity than $5^{\prime}$-GGCTAA-3' [20].

\section{Integrated data mining with differential expression levels obtained from microarray analysis}

To further investigate the transcriptional mechanisms regulated by AoXlnR, correlations between various parameters related to AoXlnR binding and the differential expression levels were analyzed (Fig. 3 and Additional files 3 and 7: Table S2 and S4). Although the peak positions detected were irrelevant for the differential expression levels of the 51 genes, the fold enrichment values, which were detected from gSELEX-Seq analysis, showed significant correlation for the differential expression level of each gene (Spearman's rank correlation coefficient = 0.419, $p<0.01$; Fig. 3a and Additional file 7: Table S4). This result indicates that the binding preference of AoXlnR identified by gSELEX-Seq correlated with the phenotype of AoXlnR analyzed by microarray. In addition, there was a significant correlation between the total number of AoXlnR-binding motifs (i.e., sum of the 5'-GGCTAG-3', 5'-GGCTAA-3', and 5'-GGCTGA-3' sites on each promoter) and the differential expression level of each gene (Spearman's rank correlation coefficient $=0.419, p<0.01$; Fig. $3 \mathrm{~b}$ and Additional file 7: Table S4). Interestingly, high correlations were showed between the differential expression level and the number of 5'-GGCTGA-3' (Spearman's rank correlation coefficient $=0.360 ; p<0.01$ ) as well as that of the major canonical binding motif, 5'-GGCTAA-3' sites (Spearman's rank correlation coefficient $=0.369 ; p<0.01$ ) (Fig. $3 \mathrm{~b}$ and Additional file 7: Table S4). The number of $5{ }^{\prime}$-GGCT GA-3' sites also showed a considerably high correlation with the differential expression level in Pearson's correlation test (Pearson's correlation coefficient $=0.519 ; p<$ 0.01; Additional file 7: Table S4). These results suggest that the 5'-GGCTGA-3' site present upstream of the gene can greatly contribute to the activation by AoXlnR in A. oryzae cells.

\section{Discussion}

AoXlnR, a TF of $A$. oryzae, is a key player in cellulose and xylan metabolisms, and activates the response of 


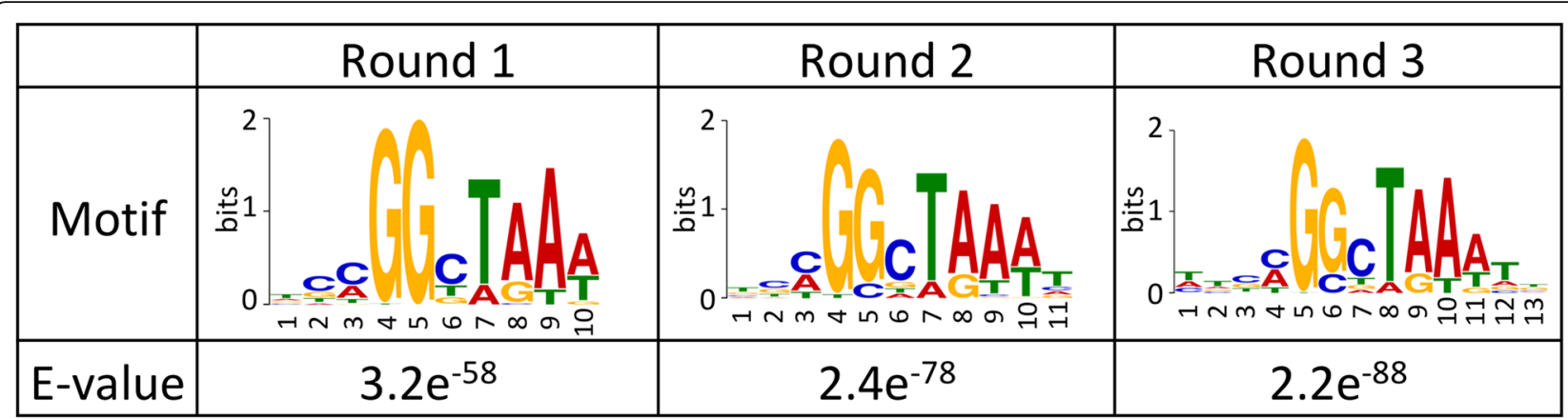

Fig. 2 Analysis of the AoXInR-binding motif. From the sequence of the peaks, 50-bp tags were extracted, and de novo motif analysis of the AoXInR-binding site with the top 100 tags ranked according to fold enrichment was performed using MEME (v 4. 10.2). Here, only motifs that showed an E-value of $<1$ are shown

xylanolytic and cellulolytic genes [20-22]. In this study, the gene expression network controlled by AoXlnR was investigated using integrated mining of gSELEX-Seq and microarray data.

By using gSELEX-Seq, 1948 promoters were identified as the candidate promoters regulated by AoXlnR (Additional file 6: Table S3). Although the canonical AoXlnR-binding motifs, 5'-GGCTGA-3' and 5' -GGCTAG-3' were present near the detected summits of the peaks in the promoters of $x \ln A$ and $c e l A$, respectively, there were no binding motifs near those upstream of $x y n F 1, x y n G 1, x y n G 2$, celB, celC, and celD (Additional file 8: Figure S4, Additional file 3: Table S2). On the other hand, the regions flanking the detected six summits contained sequences similar to the

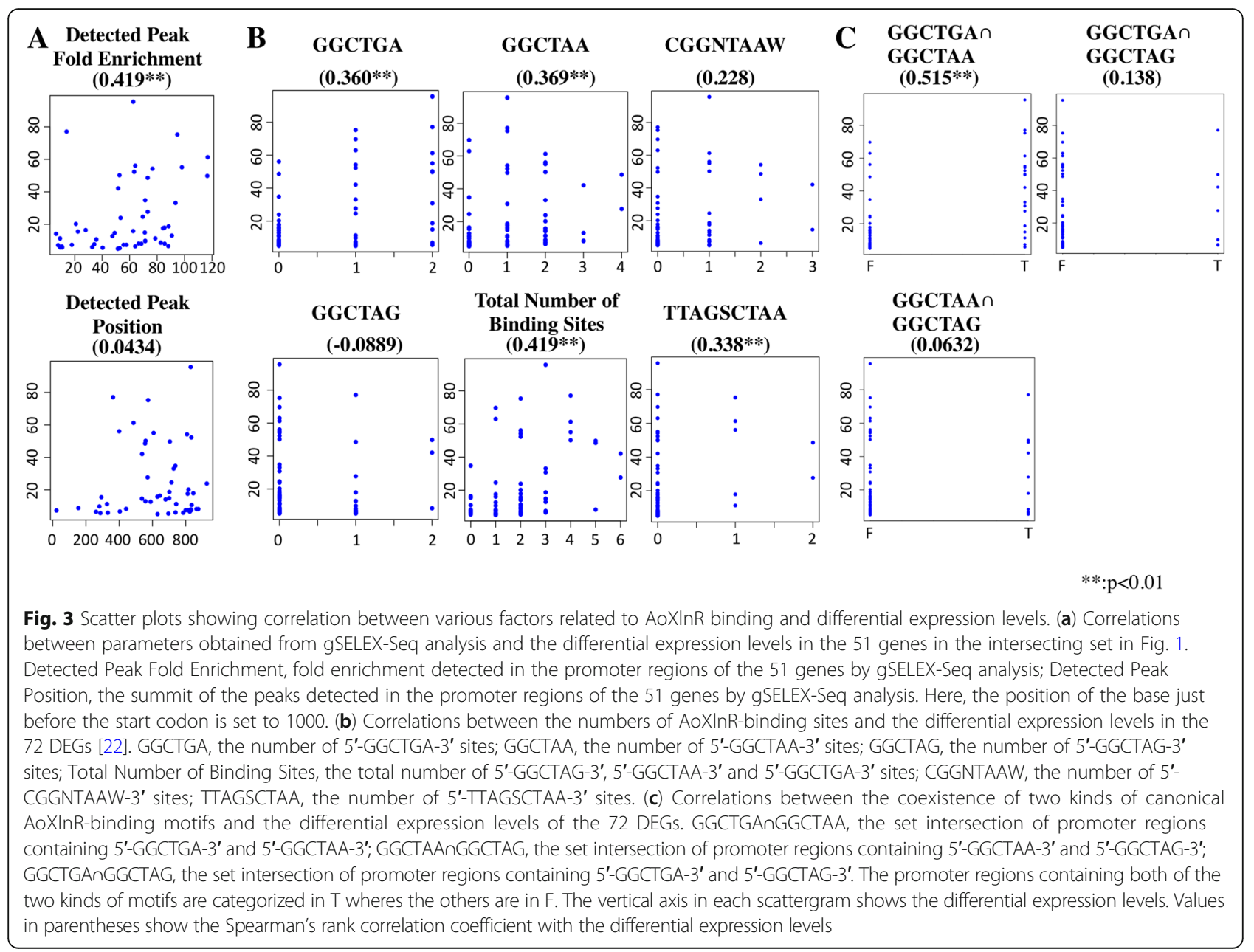


consensus motifs. These results suggest that the binding specificity of AoXlnR is relatively low, and consequently, the fragments containing sequences similar to the core motif were enriched in the gSELEX process.

To confirm the hypothesis mentioned above, the binding affinity of AoXlnR for a fragment containing the detected summit of the peak in the promoter of $x y n F 1$ was examined using BLI. The kinetic analysis indicated that xynF1_upstream_1 binds AoXlnR with higher affinity (apparent $\mathrm{KD}=311 \mathrm{nM}$ ) than XRE-WT (apparent $\mathrm{KD}=$ $656 \mathrm{nM}$ ) (Table 1). Here, xynF1_upstream_1 is a 100-bp fragment including the summit of the peak detected by gSELEX-Seq (Additional file 2: Figure S1A). Note that the 5'-GGGTAA-3' region, similar to an AoXlnR-binding motif, is present in xynF1_upstream_1 at two places (Additional file 8: Figure S4). On the other hand, XRE-WT is an AoXlnR-binding fragment retaining two AoXlnR-binding motifs (5'-GGCTAA-3' and 5'-GGCT GA-3') reported in the previous study [21] (Additional file 2: Figure S1A). It should be also noted that a minor peak near the AoXlnR-binding motif was detected in the XRE-WT region in the mapping data (Additional file 9: Figure S5). Together, these results, which AoXnlR binds with moderate affinity to a region containing no canonical binding motifs, indicated that the in vitro AoXlnR-binding specificity is relatively low. Furthermore, the results also strongly reinforce the idea that DNA fragments were preferentially enriched in accordance with their in vitro affinities for AoXlnR during the gSELEX process.

As described above, using gSELEX-Seq, 1948 promoters were identified as the candidate promoters regulated by AoXlnR (Additional file 6: Table S3). However, only 51 genes among them were included in the intersecting subset of genes identified by the gSELEX-Seq and the microarray analysis (Fig. 1). Since it is highly unlikely that AoXlnR regulates all of these genes in A. oryzae cells, we speculate that most of the other 1897 genes are false positives in gSELEX analysis. However, it is possible that the subset of the 1897 genes might include unidentified DEGs by the microarray analysis, which was conducted with mRNA extracted from mycelia that had

Table 1 Binding activity of MBP-AoXInR ${ }_{1-183}$-bound fragments

\begin{tabular}{llll}
\hline Sample ID & KD $(\mathrm{nM})$ & $\mathrm{ka}(1 / \mathrm{Ms})$ & $\mathrm{kd} \mathrm{(1/s)}$ \\
\hline xynF1_upstream_1 & 311 & $7.14 \times 10^{4}$ & $2.22 \times 10^{-2}$ \\
XRE-WT & 656 & $5.99 \times 10^{4}$ & $3.93 \times 10^{-2}$ \\
egl-242 & 40.6 & $1.24 \times 10^{5}$ & $5.04 \times 10^{-3}$ \\
egl-363 & 68.7 & $1.23 \times 10^{5}$ & $8.44 \times 10^{-3}$ \\
egl-617 & 48.4 & $9.06 \times 10^{4}$ & $4.39 \times 10^{-3}$ \\
abf-687 & 133 & $9.85 \times 10^{4}$ & $1.31 \times 10^{-2}$ \\
abf-830 & 144 & $1.54 \times 10^{5}$ & $2.22 \times 10^{-2}$ \\
\hline
\end{tabular}

been exposed to D-xylose for $30 \mathrm{~min}$ [22]. The unidentified DEGs might be revealed by RNA-Seq analysis with another AoXlnR induction condition.

In de novo motif analysis using the extracted 50-bp tags from the gSELEX-Seq, $5^{\prime}-(\mathrm{c} / \mathrm{a}) \mathrm{GGcT}(\mathrm{A} / \mathrm{g})(\mathrm{A} / \mathrm{t})(\mathrm{a} /$ t)-3' was clearly detected as an AoXlnR-binding motif (Fig. 2). Recent studies have shown that the AoXlnR monomer binds to $5^{\prime}$-CGGNTAA (A/T)-3' and the dimer to $5^{\prime}$-TTAG(G/C) CTAA-3', respectively $[28,29]$. The motif detected in Fig. 2 contained only the AoXlnR monomer-binding motif, suggesting that the recombinant AoXlnR prepared in this study, MBP-XlnR $\mathrm{R}_{1-183}$, was predominantly present as a monomer. Therefore, it may be difficult to analyze the binding sites of AoXlnR dimer using the recombinant $\mathrm{MBP}-\mathrm{X} \ln \mathrm{R}_{1-183}$. However, the association efficiency of the AoXlnR monomer might be enhanced by the attaching a protein tag, which accelerates the homodimerization of the expressed protein, to the end of the AoXlnR. We are currently planning to conduct gSELEX-Seq using AoXlnR fused with GST, which is a homodimer forming protein [30].

The results of integrated data mining with differential expression levels obtained from microarray analysis suggested that the 5'-GGCTGA-3' sites in the promoters are closely related to the regulation by AoXlnR (Fig. 3b). The promoter regions of AO090023000787 and AO090701000885, which showed significant differential expression, contain two $5^{\prime}$-GGCTGA-3' sites. Therefore, 50-bp regions containing the $5^{\prime}$-GGCTGA-3' in the two promoters were applied to BLI analysis for characterization of the AoXlnR-binding preference (Table 1 and Additional file 2: Figure S1). Interestingly, all fragments containing the binding motif analyzed were bound to AoXlnR with nanomolar affinities. The results strongly suggested that all the 5'-GGCTGA-3' sites in the promoters subject to regulation by AoXlnR.

On the other hand, using gel shift assays, Marui et al. showed that AoXlnR preferentially binds the $5^{\prime}$-GGCT AA-3' region in the $x y n F 1$ promoter with approximately 10-times higher affinity than that for $5^{\prime}$-GGCTGA-3' in the promoter [20]. In addition, they showed that the 5 '-GGCTAA-3' in the $x y n F 1$ promoter is utilized in vivo for induction by xylan and D-xylose more efficiently than the $5^{\prime}$-GGCTGA-3' site $[19,20]$. The results of these previous studies are inconsistent with our findings that the cis-element 5 '-GGCTGA-3' is significantly involved in the expression regulation by AoXlnR, comparable to 5' -GGCTAA-3' (Fig. 3b).

Of the 29 promoters possessing one or more 5'-GGCTGA-3' sites among the 72 DEGs identified by microarray analysis [22], most of them (21 promoters) also possessed 5' -GGCTAA-3' or 5' -GGCTAG-3' sites. Therefore, we analyzed the correlations between the coexistence of the two kinds of canonical AoXlnR-binding 
motifs and differential expression (Fig. 3c and Additional file 7: Table S4). The analysis showed that the presence of both the 5'-GGCTGA-3' and 5'-GGCTAA-3' sites was significantly correlated with differential expression levels (Spearman's rank correlation coefficient $=0.515, p$ $<0.01$ ). By contrast, there was no significant correlation in the case of 5'-GGCTAA-3' and 5'-GGCTAG-3' (Spearman's rank correlation coefficient $=0.0632$ ) or 5'-GGCTGA-3' and 5'-GGCTAG-3' (Spearman's rank correlation coefficient $=0.138$ ). The data mining strongly suggests that the coexistence of $5^{\prime}$-GGCTGA-3' and 5'-GGCTAA-3' sites in the promoter enhances the expression of genes located downstream of the sites. In fact, the xylan-dependent expression level of a reporter gene decreased moderately by introducing of a point mutation into the 5'-GGCTGA-3' site [20]. Together, these results suggest that the 5'-GGCTGA-3' motif might be a cis-element that further enhances the transcriptional activation by AoXlnR bound preferentially onto the 5'-GGCTAA-3' site.

The approach adopted in this study can be used for the analysis of AoXlnR-mediated cellulose and xylan metabolic network. In addition, the information of the correlation between the presence of the AoXlnR motifs and the expression of genes located downstream of the sites can be applied to $A$. oryzae metabolic engineering approaches utilizing cellulose as a starting material. Several synthetic biological approaches with a genetically engineered $A$. oryzae for cellulosic fermentation have been reported [31, 32]. In these approaches, however, several kinds of foreign cellulase genes were introduced into an $A$. oryzae transformant to enhance the cellulase activity. Considering the hypothesis described above, AoXlnR can further activate the expression of a target intrinsic cellulose gene by newly introducing the 5'-GGCTGA-3' motif into the promoter of the gene. The motif can be inserted at a desired site onto the $A$. oryzae genome by using the CRISPR/Cas9 system of filamentous fungi [33-35] to increase the cellulase activity of the strain. In principle, this strategy can be applied to various metabolite fermentations using $A$. oryzae as a cell factory. Hence, if our pipeline is combined with the CRISPR/Cas9 system, it may be possible to rationally design a metabolic process in $A$. oryzae.

Recently, Wang et al. performed a genome-wide analysis of TF-binding sites in A. oryzae by using DNase I digestion coupled with high-throughput sequencing (DNase-Seq), consequently identifying the binding site of 19 known TFs based on the digestion profile [36]. In addition, the authors found that the DNase I cleavage patterns of TFs were consistent with DNA shape features, such as minor groove twist (MGW), propeller twist (ProT), helix twist (HelT), and Roll. In the report, however, the target TF phenotypes were not considered.
On the other hand, our pipeline, which integrates both data of in vitro TF-binding characteristics and DEGs, can output not only the genes regulated directly by a TF but also the TF-binding cis-elements related closely to the expression of the target genes. A further robust transcriptome analysis system may be established by incorporating the DNA shape features described above as parameters into our pipeline.

\section{Conclusions}

In this study, the genes regulated directly by AoXlnR were successfully identified by integrated mining of data obtained from gSELEX-Seq and microarray. The data mining of the promoters of the DEGs showed that the presence of both the canonical AoXlnR-binding motifs, 5'-GGCTAA-3' and 5'-GGCTGA-3', are related significantly to the expression of genes located downstream of the sites. These findings will contribute greatly to the elucidation of AoXlnR-mediated cellulose and xylan metabolic network in A. oryzae. In addition, the pipeline, which is based on integrated mining of data consisting of both in vitro characterization of the DNA-binding sites and TF phenotype, can be a robust platform for comprehensive analysis of gene expression network via TFs.

\section{Additional files}

Additional file 1: Table S1. Oligonucleotide primers list. (DOCX 94 kb) Additional file 2: Figure S1. Promoter region used for affinity analysis. (A) The regions of XRE-WT and xynF1_upsteram_1 in xynF1Blue and red sequences indicate the regions of xynF1_upstream_1 and XRE-WT, respectively. Lower cases indicate mutation sites to derive from the designed primer. (B) The regions of egl-242, egl-363 and egl-617 are derived from promoter of AO090023000787. Blue, red and green sequences indicate the regions of egl-242, egl-363 and egl-617, respectively. (C) The regions of abf-680 and abf-837 are derived from promoter of AO090701000885. Blue and red sequences indicate the regions of of abf-680 and abf-837, respectively. AoXlnR binding motifs are shaded. Asterisks indicate the summit position of the detected peaks from the selection round 3 in gSELEX. Italic characters indicate possible AoXInR binding sites. (DOCX $213 \mathrm{~kb}$ )

Additional file 3: Table S2. Parameter list in data mining of AoXInR binding sequence. $(N)$, the number of the site; $(P)$, the posion of the site; (FE), fold enrichment. The Gene ID in gray column indicates that the peak was detected in the promoter in gSELEX-Seq analysis. (DOCX $609 \mathrm{~kb}$ )

Additional file 4: Figure S2. Construction of A. oryzae genomic library. (A) Genomic DNA shearing by using DNA-shearing system M220 (Life Technologies). (B) Gel extraction of size-fractionated genomic library after linker ligation. Bands of approximately 100-250 bp were excised using a spatula. (C) Genomic library after PCR amplification using the gel extraction product as a template. Red arrow indicates the amplicon. (TIFF 33973 kb)

Additional file 5: Figure S3. Flow cytometric analysis of selected DNA pools from gSELEX by using bead display. (A) Dot plot of fluorescence analysis. $X$-axis is quantified fluorescence intensity in the FL1 channel and $Y$-axis is quantified fluorescence intensity in FL5. (B) Relative binding affinities to $X \ln R$ was calculated by the dividing the intensity of FL 1 by that of FL5 and the binding affinity of Round 0 against AoXInR was set as 1. (TIFF 33973 kb) 
Additional file 6: Table S3. A. oryzae promoter regions selected by gSELEX-Seq. (DOCX 239 kb)

Additional file 7: Table S4. Correlation analyses between various factors related to AoXInR binding and differential expression levels. (DOC 49 kb)

Additional file 8: Figure S4. Summits of peaks detected using gSELEXSeq in the promoter regions of $x y n F 1, x y n G 1, x y n G 2, x y \mid A$, celA, celB, celC and ceID. gSELEX-Seq peaks were detected using MACS (v1.4.2). Blue squares indicate the summits of the peaks. Red square frames indicate canonical XInR binding motifs. (DOCX $278 \mathrm{~kb}$ )

Additional file 9: Figure S5. Mapping of selected DNAs in $x y n F 1$ promoter region. Sequence tags from each round were mapped on $A$. oryzae mapped with Bowtie (v2). The mapping results were visualized by Integrative Genomics Viewer (IGV). Red arrow indicates the translation initiation site. (TIFF 33973 kb)

\section{Abbreviations}

BLI: Biolayer interferometry; gSELEX-Seq: Genomic systematic evolution of ligands by exponential enrichment-Seq; MBP: Maltose-binding protein; TFs: Transcription factors

\section{Acknowledgments}

We thank Dr. Emi Kunitake of Mie University for the helpful advice on A. oryzae manipulation.

\section{Funding}

This work was supported in part by a Grant-in-Aid for Young Scientists (B) (no. 16 K18297 to TK) from the Ministry of Education, Culture, Sports, Science and Technology of Japan (MEXT). The funding body had no role in the design of the study, data collection, analysis, or interpretation of data.

\section{Availability of data and materials}

The gSELEX-Seq data analyzed in this study are available in the DNA Databank of Japan (DDBJ; accession number DRA006473)

\section{Authors' contributions}

$\mathrm{HO}, \mathrm{TK}, \mathrm{Kl}, \mathrm{HN}$, TK designed and performed the experiments. $\mathrm{HO}, \mathrm{Kl}$, TK analyzed the data and wrote the paper. All authors read and approved the final manuscript.

\section{Ethics approval and consent to participate}

Not applicable.

\section{Consent for publication}

Not applicable.

\section{Competing interests}

Not applicable.

\section{Publisher's Note}

Springer Nature remains neutral with regard to jurisdictional claims in published maps and institutional affiliations.

\section{Author details}

'Department of Applied Biosciences, Graduate School of Bioagricultural Sciences, Nagoya University, Furo-cho, Chikusa-ku, Nagoya 464-8601, Japan. ${ }^{2}$ Center for Gene Research, Nagoya University, Furo-cho, Chikusa-ku, Nagoya 464-8602, Japan

Received: 11 September 2018 Accepted: 16 December 2018 Published online: 08 January 2019

\section{References}

1. Todeschini AL, Georges A, Veitia RA. Transcription factors: specific DNA binding and specific gene regulation. Trends genet. 2014;30:211-9 Elsevier Ltd; Available from: https://doi.org/10.1016/j.tig.2014.04.002.

2. Johnston M. A model fungal gene regulatory mechanism: the GAL genes of Saccharomyces cerevisiae. Microbiol Rev. 1987;51:458-76. Available from: http://mmbr.asm.org/content/51/4/458.long
3. Marmorstein R, Carey M, Ptashne M, Harrison SC. DNA recognition by GAL4: structure of a protein-DNA complex. Nature. 1992;356:408-14 Available from: http://eutils.ncbi.nlm.nih.gov/entrez/eutils/elink.fcgi?dbfrom= pubmed\&id $=1557122 \&$ retmode $=$ ref\& $\mathrm{cmd}=$ prlinks\%5Cnpapers3:// publication/uuid/699CC99A-D2C6-4E86-AC13-8C02953A7214.

4. Schena M, Shalon D, Davis RW, Brown PO. Quantitative monitoring of gene expression patterns with a complementary DNA microarray. Science. 1995; 270:467-70 Available from: http://science.sciencemag.org/content/270/ 5235/467.long.

5. Zheng M, Wang X, Templeton LJ, Smulski DR, LaRossa RA, Storz G. DNA Microarray-Mediated Transcriptional Profiling of the Escherichia coli Response to Hydrogen Peroxide. J Bacteriol. 2001;183:4562-70. Available from: http://jb.asm.org/content/183/15/4562.long.

6. Mao J, Habib T, Shenwu M, Kang B, Allen W, Robertson L, et al. Transcriptome profiling of Saccharomyces cerevisiae mutants lacking $\mathrm{C} 2 \mathrm{H} 2$ zinc finger proteins. BMC Genomics. 2008;9:1-9. Available from: https:// bmcgenomics.biomedcentral.com/articles/10.1186/1471-2164-9-S1-S14.

7. Oliphant AR, Brandl CJ, Struhl K. Defining the sequence specificity of DNAbinding proteins by selecting binding sites from random-sequence oligonucleotides: analysis of yeast GCN4 protein. Mol Cell Biol [Internet]. 1989;9:2944-9 Available from: http://mcb.asm.org/content/9/7/2944.long.

8. Tuerk C, Gold L. Systematic evolution of ligands by exponential enrichment: RNA ligands to bacteriophage T4 DNA polymerase. Science. 1990;249:50510 Available from: http://www.ncbi.n/m.nih.gov/pubmed/2200121.

9. Ellington $A D$, Szostak JW. In vitro selection of RNA molecules that bind specific ligands. Nature. 1990;346:818-22 Available from: https://www.ncbi. nlm.nih.gov/pubmed/1697402.

10. Singer BS, Shtatland T, Brown D, Gold L. Libraries for genomic SELEX. Nucleic Acids Res. 1997;25:781-6 Available from: https://www.ncbi.nlm.nih. gov/pmc/articles/PMC146522/.

11. Dror I, Golan T, Levy C, Rohs R, Mandel-Gutfreund Y. A widespread role of the motif environment in transcription factor binding across diverse protein families. Genome Res. 2015;25:1268-80 Available from: https://genome. cshlp.org/content/25/9/1268.long.

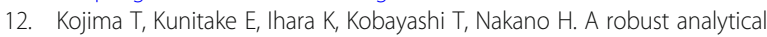
pipeline for genome-wide identification of the genes regulated by a transcription factor: Combinatorial analysis performed using gSELEX-Seq and RNA-Seq. PLoS One. 2016;11:1-16 Available from: http://journals.plos. org/plosone/article?id=10.1371/journal.pone.0159011.

13. Barbesgaard P, Heldt-Hansen HP, Diderichsen B. On the safety of Aspergillus oryzae: a review. Appl Microbiol Biotechnol. 1992;36:569-72. Available from: https://link.springer.com/article/10.1007\%2FBF00183230.

14. Beguin P. Molecular Biology of cellulose degradation. Microbiol. 1990;44:2557 Available from: https://www.annualreviews.org/doi/abs/10.1146/annurev. mi.44.100190.001251

15. van Peij NN, Gielkens MM, de Vries RP, Visser J, de Graaff LH. The transcriptional activator XInR regulates both Xylanolytic and endoglucanase gene expression in Aspergillus niger. Appl Environ Microbiol. 1998;64:3615-9. Available from: http://aem.asm.org/content/64/10/3615.long.

16. de Vries RP, Visser J, de Graaff LH. CreA modulates the XInR-induced expression on xylose of Aspergillus niger genes involved in xylan degradation. Res Microbiol. 1999;150:281-5. Available from: https://www. sciencedirect.com/science/article/pii/S0923250899800539?via\%3Dihub.

17. van Peij NN, Visser J, de Graaff LH. Isolation and analysis of XInR, encoding a transcriptional activator coordinating xylanolytic expression in Aspergillus niger. Mol Microbiol. 1998;27:131-42. Available from: https://onlinelibrary. wiley.com/doi/abs/10.1046/j.1365-2958.1998.00666.x.

18. Gielkens MM, Dekkers E, Visser J, de Graaff LH. Two Cellobiohydrolaseencoding genes from Aspergillus niger require D-xylose and the Xylanolytic transcriptional activator XInR for their expression. Appl Environ Microbiol. 1999;65:4340-5. Available from: http://aem.asm.org/ content/65/10/4340.long.

19. de Vries RP, van de Vondervoort PJ, Hendriks L, van de Belt M, Visser J. Regulation of the a-glucuronidase-encoding gene ( aguA ) from Aspergillus niger. Mol Genet Genomics. 2002;268:96-102. Available from: https://link. springer.com/article/10.1007\%2Fs00438-002-0729-7.

20. Marui J, Kitamoto N, Kato M, Kobayashi T, Tsukagoshi N. Transcriptional activator, AoXInR, mediates cellulose inductive expression of the xylanolytic and cellulytic genes in Aspergillus oryzae. FEBS Lett. 2002;528:279-82. Available from: https://febs.onlinelibrary.wiley.com/doi/abs/10.1016/S00145793\%2802\%2903328-8. 
21. Marui J, Tanaka A, Mimura S, de Graaff LH, Visser J, Kitamoto N, et al. A transcriptional activator, AoXInR, controls the expression of genes encoding xylanolytic enzymes in Aspergillus oryzae. Fungal Genet Biol. 2002;35:157-69. Available from: https://www.sciencedirect.com/science/article/pii/ S1087184501913210?via\%3Dihub.

22. Noguchi Y, Sano M, Kanamaru K, Ko T, Takeuchi M, Kato M, et al. Genes regulated by AoXInR, the xylanolytic and cellulolytic transcriptional regulator, in Aspergillus oryzae. Appl Microbiol Biotechnol. 2009;85:141-54. Available from: https://link.springer.com/article/10.1007\%2Fs00253-009-2236-9.

23. Kojima T, Hashimoto $Y$, Kato M, Kobayashi T, Nakano H. High-throughput screening of DNA binding sites for transcription factor AmyR from Aspergillus nidulans using DNA beads display system. J Biosci Bioeng. 2010; 109:519-25. The Society for Biotechnology, Japan; Available from: https:// doi.org/10.1016/j.jbiosc.2009.11.024.

24. Bradford MM. A rapid and sensitive method for the quantitation microgram quantities of protein utilizing the principle of protein-dye binding. Anal Biochem. 1976;254:248-54 Available from: https://www.sciencedirect.com/ science/article/pii/0003269776905273?via\%3Dihub.

25. Cerqueira GC, Arnaud MB, Inglis DO, Skrzypek MS, Binkley G, Simison M, et al. The Aspergillus Genome Database: multispecies curation and incorporation of RNA-Seq data to improve structural gene annotations. Nucleic Acids Res. 2014;42(database issue):D705-10.https://www.ncbi.n/m. nih.gov/pubmed/24194595.

26. Concepcion J, Witte K, Wartchow C, Choo S, Yao D, Persson H, et al. Label-free detection of biomolecular interactions using BioLayer interferometry for kinetic characterization. Comb Chem High Throughput Screen. 2009;12:791-800 Available from: https://www.ncbi. nlm.nih.gov/pubmed/19758119.

27. Tamano K, Sano M, Yamane N, Terabayashi Y, Toda T, Sunagawa M, et al. Transcriptional regulation of genes on the non-syntenic blocks of Aspergillus oryzae and its functional relationship to solid-state cultivation. Fungal Genet Biol. 2008;45:139-51. Available from: https://www.ncbi.nlm.nih.gov/pubmed/17967552.

28. Kunitake E, Kobayashi T. Conservation and diversity of the regulators of cellulolytic enzyme genes in Ascomycete fungi. Curr genet. 2017;63:951-8. Berlin: Springer; Available from: https://link.springer.com/article/10. 1007\%2Fs00294-017-0695-6.

29. Ishikawa K, Kunitake E, Kawase T, Atsumi M, Noguchi Y, Ishikawa S, et al. Comparison of the paralogous transcription factors AraR and XInR in Aspergillus oryzae. Curr genet. 2018;64:1245-60. Berlin: Springer; Available from: https://doi.org/10.1007/s00294-018-0837-5.

30. Nishida M, Harada S, Noguchi S, Satow Y, Inoue H, Takahashi K. Threedimensional structure of Escherichia coli glutathione S-transferase complexed with glutathione sulfonate: catalytic roles of Cys10 and His106. J Mol biol. 1998;281:135-47 Available from: https://www.ncbi.nlm.nih.gov/ pubmed/9680481.

31. Lin H, Wang Q, Shen Q, Ma J, Fu J, Zhao Y. Engineering Aspergillus oryzae A4 through the chromosomal insertion of foreign cellulase expression cassette to improve conversion of cellulosic biomass into lipids. PLoS One. 2014;9:e108442. Available from: http://journals.plos.org/plosone/article?id= 10.1371/journal.pone.0108442.

32. Yamada R, Yoshie T, Wakai S, Asai-Nakashima N, Okazaki F, Ogino C, et al. Aspergillus oryzae-based cell factory for direct kojic acid production from cellulose, Microb Cell Fact. 2014;13:71. Available from: https:// microbialcellfactories.biomedcentral.com/articles/10.1186/1475-2859-13-71.

33. Nødvig CS, Nielsen JB, Kogle ME, Mortensen UH. A CRISPR-Cas9 system for genetic engineering of filamentous fungi. PLoS One. 2015;10:1-18 Available from: http://journals.plos.org/plosone/article?id=10.1371/journal.pone.0133085.

34. Katayama T, Tanaka Y, Okabe T, Nakamura H, Fujii W, Kitamoto K, et al. Development of a genome editing technique using the CRISPR/Cas9 system in the industrial filamentous fungus Aspergillus oryzae. Biotechno Lett. 2016;38:637-42. Available from: https://link.springer.com/article/10. 1007\%2Fs10529-015-2015-x.

35. Nakamura H, Katayama T, Okabe T, Iwashita K, Fujii W, Kitamoto K, et al. Highly efficient gene targeting in Aspergillus oryzae industrial strains under ligD mutation introduced by genome editing: Strain-specific differences in the effects of deleting $E c d R$, the negative regulator of sclerotia formation. J Gen Appl Microbiol. 2017;63:172-8. Available from: https://www.jstage.jst.go. jp/article/jgam/63/3/63_2016.10.002/_article.

36. Wang C, LV Y, Wang B, Yin C, Lin Y, Pan L. Survey of protein-DNA interactions in Aspergillus oryzae on agenomic scale. Nucleic Acids Res. 2015;43:4429-46. Available from: https://academic.oup.com/nar/article/43/9/4429/1128438.

\section{Ready to submit your research? Choose BMC and benefit from:}

- fast, convenient online submission

- thorough peer review by experienced researchers in your field

- rapid publication on acceptance

- support for research data, including large and complex data types

- gold Open Access which fosters wider collaboration and increased citations

- maximum visibility for your research: over $100 \mathrm{M}$ website views per year

At $\mathrm{BMC}$, research is always in progress.

Learn more biomedcentral.com/submissions 